\title{
Transmission Electron Microscopy and Three-Dimensional Tomography of Peptide-Coated
} \section{Single-Walled Carbon Nanotubes}

\author{
P. Bajaj,* J. Nguyen,* C. Gilpin,** G.R. Dieckmann,* C.C. Chiu, ${ }^{*}$ S.O. Nielsen, ${ }^{*}$ and I.H. \\ Musselman*
}

*Department of Chemistry, The University of Texas, Dallas, Richardson, TX 75080

**Department of Cell Biology, UT Southwestern Medical Center, Dallas, TX 75390

Transmission electron microscopy (TEM), combined with tomography and three dimensional (3-D) reconstruction techniques, was used to investigate the structural organization of a designed polypeptide which self-assembles on the surface of single-walled carbon nanotubes (SWNTs). The amphiphilic helical peptide nano-1 [1], which has previously been shown to noncovalently coat and disperse hydrophobic SWNTs in aqueous environments, was utilized as a model system in this study. While the dispersal ability of these peptides has been demonstrated [2, 3], we still need to understand the structure and orientation of these peptides on the SWNT surface. This knowledge is critical for the future design of peptides exhibiting enhanced stability and efficient coating of SWNTs.

Using FEI Inspect 3-D tomography data collection software, a series of tilt images was acquired from $-60^{\circ}$ to $+60^{\circ}$ at 1 or 2 degree increments using a Fischione high tilt sample holder at $68,000 \times$ magnification $(0.2 \mathrm{~nm}$ per pixel). For image acquisition, the peptide-coated nanotube was maintained along the $\mathrm{Y}$-axis suspended across the lacey grid. The microscope was corrected for eucentric height, and direct alignments were performed including full gun alignment, $\mathrm{X}$ and $\mathrm{Y}$ pivot points, beam shift, and rotation center. Gold nanoparticles (Ted Pella, size $=5$ or $10 \mathrm{~nm}$ ) were introduced as fiducial markers. The gold colloid $(5 \mu \mathrm{L})$ was dropped onto a glow discharged lacey carbon grid and the excess was wicked away after $2 \mathrm{~min}$. The nano-1/SWNT dispersion was diluted 1:10 and $1 \mu \mathrm{L}$ was dropped on top of the gold nanoparticles (Figure 1d). Data sets were aligned using IMOD [4] and volume reconstructions compared using either weighted back projection (WBP) in IMOD or a simultaneous iterative reconstruction technique using a FEI Express 3D GPUaccelerated workstation. Automated tomography relies on auto focusing capabilities of the software in acquiring 2-D projections of the tilt series [5]. Auto-focusing allows optimum focusing at low tilt angles, but, at high angles, tilt induced differences in sample height and the location of gold fiducials limits the auto-focusing. As a result, auto-focusing variations occur as the dense gold spheres form the basis for focusing. In order to address variations in auto focusing, a focal series was acquired at each tilt angle. The focal series chosen consisted of a set of images taken at $50 \mathrm{~nm}$ defocus steps ($0.05,-0.10,-0.15,-0.20,-0.25$, and $-0.30 \mu \mathrm{m})$.

In summary, the results were compared to atomic force microscopy images obtained from dried samples, traditional TEM images, and molecular dynamics computer simulations (Figure 1a, b and c). It is interesting to note the difference in the information contained in the thin computed z-slice post processing. The image information in the computed 3-D z-slice (Figure $2 \mathrm{~b}$ ) is not averaged throughout the 3-D volume and thus is more interpretable compared to a 2-D slice (Figure 2 a) which appears cluttered with information superimposed. SIRT scheme offers an iterative method for blurring or graininess correction involving a large number of simultaneous iterations to compute the final reconstructed 3-D volume and provided better contrast restoration than WBP (Figure $2 \mathrm{~b}$ and c). Acquiring a tomography tilt series in conjunction with the focal series option allowed a data set to be constructed from the most appropriately focused images at each tilt angle (Figure $3 \mathrm{a}, \mathrm{b}, \mathrm{c}$ ). 3$\mathrm{D}$ visualization was performed using computational slicing and surface rendering of the 3-D volume. 2-D slices were made transversely or longitudinally in the direction perpendicular to the viewing direction. These digital 2-D images were viewed as XY, XZ or YZ slices (Figure 4a, b). The image 
information in digitally sliced images is not averaged over the entire volume and allows better contrast and more accurate interpretation in comparison to the full 2-D projection. The intensity range of the sample features were measured based on an intensity plot feature in the data analysis module (Figure 4a). This range is applied to the 3-D volume by 'thresholding' to distinguish real data information from noise and clustered regions in the slice and 'contour mapping' (Figure 4a, b). As a result, a surface rendered 3-D model of the peptide/SWNT assembly was viewed (Figure 4c).

References

[1] G.R. Dieckmann et al., J. Am. Chem. Soc. 125 (2003) 1770.

[2] V. Zorbas et al., J. Am. Chem. Soc. 126 (2004) 7222.

[3] V.Z. Poenitzsch, I.H. Musselman, Microsc. Microanal. 12 (2006) 221.

[4] J.R. Kremer et al., J. Struct. Biol. 116 (1996) 71.

[5] A.J Koster et al., Ultramicrosc. 46 (1992) 207.

[6] This work was supported by the Human Frontier Science Program (GRD).


Figure 1. (a) AFM image of peptide/SWNT dispersion. (b) TEM image of peptide-coated SWNT on lacey carbon grid. (c) Molecular dynamics simulation of peptide organization on SWNT surface showing a bundle of 5 nano-1 peptides around a SWNT (radius $=4.1 \AA$ ). (d) TEM image of peptide/SWNT dispersion on lacey grid. Gold nanoparticles $(10 \mathrm{~nm})$ were introduced as fiducial markers for fine alignment of tomography tilt series.

Figure 2. (a) A single 2-D TEM projection of a peptide-coated SWNT from raw aligned tilt series image. A single z-slice from a 3-D volume reconstruction performed by WBP using (b) IMOD and (c) SIRT algorithms for restoration and enhancement of image contrast.

Figure 3. Focal series of peptide-coated SWNTs. Images (a) to (c) show an acceptable focal range encompassing defocus values (shown here at $-0.10,-0.15$, and $-0.20 \mu \mathrm{m}$ ) in increments of $50 \mathrm{~nm}$ defocus steps. Image (a) shows an under focused image, (b) optimum focus, and (c) over focused.

Figure 4. (a) A z-slice (longitudinal) and (b) y-slice (transverse) from the 3-D volume as seen through ParaView. The white line in (a) indicates the region over which the intensity range (inset) was plotted. Contour plot of (b) a single 2-D z-slice, and a y-slice encompassing the defined threshold range. (c) Surface rendered 3-D model of the peptide-coated SWNT. 